University of Nebraska - Lincoln

DigitalCommons@University of Nebraska - Lincoln

2001

\title{
Technology as the Representative Anecdote in Popular Discourses of Health and Medicine
}

Lynn M. Harter

Department of Speech Communication and Theatre Arts, Minnesota State University, Moorhead

Phyllis Japp

University of Nebraska - Lincoln, pjapp1@unl.edu

Follow this and additional works at: https://digitalcommons.unl.edu/commstudiespapers

Part of the Communication Commons

Harter, Lynn M. and Japp, Phyllis, "Technology as the Representative Anecdote in Popular Discourses of Health and Medicine" (2001). Papers in Communication Studies. 2.

https://digitalcommons.unl.edu/commstudiespapers/2

This Article is brought to you for free and open access by the Communication Studies, Department of at DigitalCommons@University of Nebraska - Lincoln. It has been accepted for inclusion in Papers in Communication Studies by an authorized administrator of DigitalCommons@University of Nebraska - Lincoln. 


\title{
Technology as the Representative Anecdote in Popular Discourses of Health and Medicine
}

\author{
Lynn M. Harter \\ Department of Speech Communication and Theatre Arts \\ Minnesota State University, Moorhead \\ Phyllis M. Japp \\ Department of Communication Studies \\ University of Nebraska
}

\begin{abstract}
Using a Burkean framework (1969), this article approaches medical dramas as cultural texts to be read for dominant meanings of health and health care. Burke's representative anecdote illuminates the melding of science, technology, and healing in popular discourses of health, establishing technological intervention as the norm and marginalizing nontechnological (i.e., alternative) forms of health care. Popular entertainment reinforces this anecdote in narratives of healing as technological competence triumphing over nature.
\end{abstract}

The role of mass mediated messages in shaping our knowledge and understandings of health and disease has captured the attention of our government and health communication scholars (e.g., Clarke, 1992; Glik et al., 1998; Parrott, 1996; Vanderford, 1999). In 1982, the Surgeon General's Scientific Advisory Committee issued a report on TV in our culture, including a discussion of the medium's health-promoting possibilities (U.S. Department of Health and Human Services, 1982). Among other imperatives, the committee issued a call for more research about "the content of television programs and portrayals relevant to health" (U.S. Department of Health and Human Services, 1982, p. 9). Since 1982, researchers have examined indirect and direct health messages embedded within programming to better understand constructed images of health and their societal effects (e.g., Larson, 1991; Pfau, Mullen, \& Garow, 1995; Sharf \& Freimuth, 1993; Turow \&

Requests for reprints should be sent to Lynn M. Harter, Department of Speech Communication and Theatre Arts, Minnesota State University, Moorhead, MN 56563. 
Coe, 1985). This article contributes to this line of inquiry by rhetorically approaching medical dramas as cultural texts to be read for dominant meanings of health and healing, focusing particular attention on how the technological imperative is instantiated and reinforced in this discourse.

Popular entertainment is increasingly acknowledged as a source of understanding about how social institutions, including health services, are structured (Storey, 1993; Tulloch \& Lupton, 1997). Although we recognize cultural artifacts as presenting either fictious or heavily dramatized accounts of real incidents, we nevertheless are susceptible to the definitions of reality, value systems, power structures, and role relations we see imaged again and again in our entertainment. As suggested by Vanderford (1999), "Television, as the dominant form of mass media, is an important source of shared perceptions which offers lessons for viewers about the way the world works" (p. 33). When faced with a problem or crisis, these impressions are part of our repertoire of knowledge. When defining health, or confronting illness, we certainly may consult the appropriate scientific practitioners; however, intermingled with their advice are the images and impressions from popular news, books, films, and TV. Therefore, our knowledge about health, illness, prevention, and treatment is constructed from these various, and often contradictory, bits and pieces of information amassed from everyday activities of our lives.

The institution of medicine has long been a favorite subject for TV drama, with Dr. Welby, Trapper John, St. Elsewhere, Chicago Hope, ER, and LA Doctors only a few examples. Although most viewers tune in to shows like ER and Chicago Hope for the drama rather than the medicine, "it's hard not to pick up a little medical lore along the way" (Barnes \& McDowell, 1998, p. 74). Commenting on the impetus for the ever-rising popularity of medical dramas, Zurawik (1999) pointed to our culture's obsession with health consciousness combined with widespread anxiety about health care delivery in health maintenance organizations and other forms of managed care. Medical foundations are aware of the widespread appeal and potential persuasive impact of medical dramas and as reported in an April 1998 article in the Wall Street Journal (McGinley, 1998), such groups have been able to successfully lobby these shows to include dramatizations of specific diseases. Likewise, scholars and public health officials are working with producers of entertainment TV to develop programs that incorporate intervention strategies for public health education and promotion purposes (e.g., Glik et al., 1998).

This article examines the 1997 through 1998 and 1998 through 1999 seasons' episodes of Chicago Hope and ER. We seek to identify the overall vision of western institutional medicine as presented in these dramas. After viewing all of the episodes, we located several primary and interlocking themes we believe to be dimensions of the representative anecdote of technology. We selected short examples that clearly demonstrate the theme under discussion. We are not arguing that it is the only, or best, reading of this discourse. Critics operating from within a different perspective or with a different focus may read the dramas quite differently (e.g., Vanderford, 
1999). We do not claim to have discovered the only important issues related to technology and medicine, rather, we have attended to one small portion of one TV genre and attempted to discover how the assumptions and expectations of technology are displayed. We propose that medical dramas serve to reinforce the dominance and legitimacy of technological medicine even as they at times appear to question its power.

\section{CRITICAL ORIENTATION}

Burke's (1969) theory of symbolic action, often called dramatism, is familiar to many scholars in communication, rhetoric, media studies, sociology, psychology, and political science. His major premise can be stated as follows: Humans "act," that is, they create social worlds through their use of language and other symbol systems. The choice of symbols to describe human desires, emotions, situations, conflicts, contexts, and all other psychosocial-material phenomena reveals not only how those phenomena are perceived and understood but how humans will act toward them. Therefore, symbolic constructions become our realities, the baselines we take for granted as we maneuver through our worlds of experience. For Burke, human motivation is not extrasymbolic (i.e., buried in the hidden psyche), but rather is located in these sense-making and action-orienting patterns displayed in discourse. By careful analysis of the discourse surrounding an idea or situation, then, we can uncover basic assumptions that shape and limit the choices of action available in that situation.

Burke (1969) provided a variety of critical concepts with which to interpret and analyze the discourses constructed and maintained by human agents in given situations. For example, symbolic discourses reveal the source or force of action, the agent; the nature of action, the act; the context in which action takes place, the scene; the manner or means of action, the agency; and the ultimate goals that propel the action, the purpose. These five terms comprise Burke's best-known critical schema, the dramatistic pentad, a conceptual framework frequently employed by critics. Certainly, a pentadic reading of medical dramas would prove insightful; however, we wish to employ a different Burkean conceptual "umbrella" in our analysis-the representative anecdote. ${ }^{1}$

\footnotetext{
${ }^{1}$ We acknowledge the value of considering medical personnel as agents, the ER as scene, technology as agency, healing as act, and so on. Certainly, the use of technology could be well served by an exploration of the tensions or ratios between agents and agency. If pushed to assign technology to a pentadic term, we would probably argue that it is "scenic" (i.e., that it is the substance and ground of perception, interpretation, and action, and thus defines the acts and agents in accordance with its characteristics). However, we believe that defining technology as representative anecdote captures more accurately the essence of the pervasive power and promise of technology, and how it has shaped our understandings of health, illness, the body, physicians, diagnosis, and treatment. Even in challenge, the power of the anecdote is recognized. Therefore, although some may operate outside of the anecdote, they are marginalized and required to present justifications for their rejection of the assumptions encoded into the anecdote.
} 
We suggest that technology, in medical dramas as in the institutions of modern medicine, is not merely the agency or tool of agents (i.e., a set of techniques that can be employed at will). We believe, with Burke (1969), that technology embraces more than mechanical "tools of the trade" or optional interventions to be used at the discretion of medical professionals. Rather, in the modern institutions of science and medicine, technology is a paradigmatic frame of reference-a way of thinking, talking, and acting that incorporates training, hierarchies, processes, and values as well as the instruments developed within that paradigm. Technology's attendant attitudes and realities so pervade Western medical practice and are so integral to perception, orientation, hierarchy, and action that it cannot be conceived as under the control of human agents. Whether medical professionals and their patients opt for technological instrumentation or "natural" healing, choices are defined and enacted firmly within the symbolic framework of technological dominance even when agents may reject a particular technological tool at a given moment.

Among Burke's (1969) many concepts for locating symbolic pattens is what he called the representative anecdote. Burke defined a representative anecdote as an idea that is both representative (i.e., broad enough to be diffused throughout the discourse in question), yet reductive (i.e., the essence of the discourse is condensed into a concise and readily understood equation). For example, "drama" is Burke's representative anecdote for human behavior. According to Brummett (1984), "[a]n anecdote is a dramatic form which underlies the constant or the specific vocabulary of discourse" (p. 162). In other words, representative anecdotes are consistent themes and stories that are sufficiently broad so as to encompass the general qualities of the discourse including its dialectical tensions and oppositions. When one engages in the search for a representative anecdote, he or she is looking for a stable form or set of relations that pervade a discourse, one that appears and reappears in different guises or variations on a theme. Therefore, a representative anecdote reveals the fundamental characteristics of a discourse and fuses its essential values.

Scholars using the representative anecdote to analyze contemporary discourse have advanced perspectives on how this tool can be used within media criticism. For instance, Brummett (1984) argued that the representative anecdote is a lens, filter, or template through which a critic studies and reconstructs the discourse. Subsequently, the critic represents the essence of the discourse by viewing it as if it follows a dramatic plot. Japp (1991) extended this position by arguing that the representative anecdote is "a core idea or equation that can be developed narratively in various ways, some of which appear contradictory rather than as containers whose content can be concretely defined" (p. 52). When used as a critical perspective, the representative anecdote allows for identification of patterns across general forms of discourse, highlights the relation of discourse to its cultural context, and identifies the dominant values of an era as depicted in the discourse in question (Japp, 1991). In other words, the representative anecdote allows critics to go be- 
yond surface narratives or scripts and tap into deeper, more implicit themes that shape the discourse. Brummett (1984) argued:

To identify a representative anecdote as immanent within discourse is to sum up the essence of a culture's values, concerns, and interests in regard to some real life issues or problems-in television, anecdotes combine visual and verbal imagery to condense prevailing attitudes and understandings. (p. 164)

A representative anecdote establishes parameters, norms, and hierarchies while developing and reinforcing language and other symbol systems that will operate within its boundaries. As such, the representative anecdote is especially valuable for a critical analysis of the implicit assumptions and values held by a particular culture as manifested in that culture's mediated texts. It is important to remember that a representative anecdote is not necessarily monolingual (Japp, 1991), rather it can and does incorporate essential tensions and conflict within the discourse in question. Therefore, those that question or challenge some aspects of the anecdote (e.g., its hierarchies) must use the language of and work within the frameworks established by the anecdote. Those who choose to reject the dominant anecdote must continually present justifications for their position, again using the language and terms of the anecdote. We believe that considering technology as the representative anecdote in medical dramas captures the pervasive power and promise of technology and how it has shaped our understandings of health, illness, the body, medical professions and institutions, as well as diagnosis and treatment of patients.

\section{THE TECHNOLOGY ANECDOTE IN MEDICAL DRAMAS}

Certainly, medical dramas play out our cultural ambivalence about technology. Technology, inspiring both fear and faith, is commonly understood as both a destroyer and savior of human life. Given this historical ambivalence, technology must continually be sold, its benefits reiterated, beliefs in its beneficence reinforced, and fears mitigated. Therefore, each new technological development requires not only the legitimacy accorded by scientific authorities but also the services of advertisers and popularizers. The public must be enticed to believe in processes and techniques that are opaque to common sense, developed, administered, and fully understood only by a priesthood of initiates. For example, as reproductive technology "progresses" into genetic engineering, public hope and fear mingle and must be "managed" by those who wish to introduce the new technology. This cultural complex of acceptance and rejection of technology is the context within which medical dramas operate persuasively.

In U.S. medicine, the technological orientation is encapsulated in the metaphor of "body as machine," a way of perceiving and defining illness as a malfunction of one 
of the "parts," and health as the intervention and restoration of that part to "working order." The metaphor incorporates a terminology or language, legitimates a corps of professionals and practitioners, establishes hierarchies among those legitimated, supports and maintains administrative structures, processes, and techniques as part of the technology of health care. Medical dramas such as ER and Chicago Hope are conceptualized and enacted within the aforementioned metaphor.

Geographically, the setting of both ER and Chicago Hope is a large urban hospital. ER is portrayed as the county hospital, whereas Chicago Hope is portrayed as an upscale research-oriented hospital. Despite their differences, technology structures the action in both dramas. In both, patients usually enter the hospital in critical conditions either because of natural ailments or trauma at which point all of the hospital's technological prowess is brought to bear on the patient. The life or death of the patient hangs in the balance as doctors attempt to devise strategies for harnessing the full potential of available technologies for healing the patient.

Certainly, ER and Chicago Hope attempt to depict technology as neutral and benign instrumentation responsive to the control of human agents, agents whose moral choices will determine whether those instruments are to be used for good or ill. Therefore, the shows are filled with scenes defining technology as a choice (e.g., "should we perform surgery?"), rather than as the overarching anecdote or paradigm of health care. However, with each of the themes we identify later, we attempt to reach beyond the premise of "technology as tool" and tap into the unspoken expectations and assumptions within which those scripts are embedded.

The technological imperative is immediately evident in these dramas, visually as well as verbally. A typical episode of $E R$, for example, begins with a patient being brought to the emergency room, usually via ambulance, after suffering some critical trauma. On entering the ER, cameras highlight the patient being tossed from one gurney to another as doctors are given the "bullet" (i.e., patient history) by the paramedics or family members. The doctors immediately begin issuing commands consisting of once foreign but now familiar terminology like "CBC," "cross type and match," "ready an intubation tray," and "start an IV flow with normal saline." As the doctor issues these orders, other staff are hooking the patient up to machines with various tubes and wires all while the heart monitor in the background provides an auditory reminder of the patient's status. Taken together, these images, sounds, practices, roles, and rules reflect the normalcy and dominance of the technological anecdote.

Whether the specific storyline refers to a state-of-the-art laser microsurgery to remove a vascular tumor from a patient's spine or an intervention as simple as antibiotic eye drops, the representative anecdote of medical dramas reflects the ideological assumption that medicine is first and foremost about disease. The persuasive visual and verbal constructions not only reflect societal perceptions of science and technology but also serve to naturalize the technological anecdote as they create the illusion that technology and health care are one in the same. As the 
story proceeds, patients serve as a foil for demonstrating the efficiency and power of modern technological medicine as introduced in the opening sequences.

In the following, we highlight three major themes that we believe comprise the representative anecdote of technology in medical dramas: technology's dominance over nature, healing as technological competence, and technology's marginalizing of alternative medicine. These themes are interlocking and mutually reinforcing elements of the anecdote of technology. As we point out, at times scripts may challenge each of these themes. The challenge is easily contained, however, and deflected by the other themes and by the overarching paradigm of technology.

\section{Technology Triumphs Over Nature}

A central theme of technology in medical dramas is the ongoing conflict between nature and technology. Nature, in earlier times a dominant paradigm of health and disease, has been displaced by technology. In the technological paradigm, disease is a product of nature, whereas technology is the force that can conquer nature, cure disease, eliminate pain and suffering, and triumph over death. Therefore, medical dramas reflect this consistent theme of the superiority and power of technology, as its heroes battle the diseases and perils inherent in the natural condition.

In one episode of Chicago Hope, two of the doctors, Kronk and Grad, are married and ready to have their first child. While attending a lamaze class, Dr. Kronk repeatedly challenged the instructor on "medical facts" concerning natural childbirth. For instance, Kronk interrupted the instructor when she was talking about why many women want to engage in the natural childbirthing processes:

I've seen this a hundred times ... . Women come into the hospital and they want natural childbirth. They get to maybe six or seven centimeters and they start screaming for an epideral. And, they start screaming at us- the doctors-as if natural childbirth was our idea.

In a statement that succinctly summarizes all three of the themes, Kronk equates nature with pain, establishes his legitimacy as a technological expert, and marginalizes natural childbirth. Comfortable in the hierarchy established by technology, Kronk exemplifies how childbirth has become a medical condition, not a natural event.

Natural childbirth is far from natural; within the technological paradigm it is a somewhat suspect practice that, if approved and controlled by technology, might be acceptable in certain cases. As Treichler (1989) noted

Obstetrics has been successful—not in creating safe childbirth but in creating a monopoly. For its own professional gain, organized medicine in the United States has 
"medicalized" childbirth, a process that in other cultures and other countries frequently takes place outside hospitals with little medical intervention. (p. 425)

The technologizing or medicalizing of a natural process such as birth means that childbirth is defined as a pathology to be treated rather than a natural process to be appreciated. Here we see an excellent example of how even the choice to "go natural" is invested with the assumptions and implications of the technological imperative and undertaken against the skepticism of doctors who may believe the technological option is superior to the natural alternative. It is interesting to note that this storyline climaxed when the doctors chose the natural childbirthing option until complications arose and an emergency caesarean operation was performed. Technology was waiting in the wings and able to rescue the mother and baby from the dangers of nature.

In another example of technological dominance, an episode of $E R$ provided viewers an opportunity to explore tensions between the promise of technology and the natural condition of aging. An elderly man was brought to the emergency room because he was suffering from dehydration and malnutrition. After examining the patient, Dr. Carter, an aspiring young resident, concluded that the man was suffering abuse in the form of neglect from his family caregivers and needed full-time medical attention provided by a primary care institution. The man pleaded to not receive full-time medical attention and treatment: "I am 86 years old. I've slept in the same bed for 70 years. I just want to die in my own bed."

As with childbirth, technological medicine medicalizes and pathologizes the natural condition of aging and the inevitable occurrence of death. Goodwin (1999) argued that elderly patients "serve as a mirror, reflecting the limitations and sometimes the absurdities of modern medicine" (p. 1283). The very old are viewed as challenges to technological dominance and are often imprisoned in technological medicine's insistence that they be diagnosed, treated, and their life prolonged whether they desire such or not. An 86-year-old man in ill health will die. In the language of technological medicine, however, he must be "allowed" to die (i.e., technology must give permission for nature to take its course). Neither do the patient's wishes appear to have legitimacy in the technological paradigm. This episode concluded with Dr. Carter implementing the medical solution rather than giving the patient his desire. The state assumed custody of the patient and admitted him into the nursing home where "he can receive constant medical attention."

A final example of how the discourse of medical dramas often pits technology versus nature was an ongoing storyline in $E R$ where an attending doctor's son, Scott, was diagnosed with leukemia. This storyline was particularly powerful because it involved a young child's battle with cancer. Scott and his father were faced with the decision of entering an experimental Phase-1 chemotherapy program after no bone marrow donors were found. Initially, the family decided to participate in the research project even though it posed great risk to the child. During this time, several staff 
members called the child "brave" and characterized him as "tough" and a "real fighter." With state-of-the-art technological weapons, Scott and his family were ready to combat the natural enemy of disease. At one point, the side effects of the chemotherapy resulted in the child going into cardiac arrest. During resuscitation efforts, one physician commented in frustration, "What is it with these parents-why won't they just let these kids go?" Ultimately, due to the harsh side effects of the treatment, the child withdrew from the study. He died shortly thereafter.

Scott's story in $E R$ appears to challenge the powers of technological medicine and even suggests that technology can cause as well as alleviate suffering. However, notice that Scott's choice to accept experimental treatment was viewed positively by doctors and his family. For accepting the risks of treatment, Scott was labeled "courageous." Looking closely at this episode, even in challenge the dominance of technology is implicitly reinforced. When victory is not achieved, as in this case, the technological recourse is to explain the lost battle as one step in winning the war. Short-term losses are necessary for long-term gains, but it is taken for granted that technology will ultimately triumph.

Scripts in medical dramas perpetually attempt to ease tensions between technology and nature, yet continue to equate disease and death with nature and healing with technology. Implicitly, nature is the enemy and technology is the savior. Even when technology appears to be merely a tool of choice, as in the earlier examples, one must reflect on how technology has redefined the conditions exhibited — childbirth, aging, death, and so forth—as medical conditions requiring professional treatments. An important implication of the technological anecdote is the medicalizing of all life processes, thus bringing them under the control and surveillance of technological medicine. Whether the professionals — or the patients — choose to intervene with technological tools or "let nature take its course," the choices are explored in the language of technological medicine, with full awareness of the power of technology to determine who is party to the conversation. The prevailing attitude of medical professionals that nature is the enemy is exemplified by a comment made by a Chicago Hope physician treating a family for kidney failure due to organically grown vegetables: "This is exactly why I have never been into the back to nature thing: Give me additives and preservatives any day."

\section{Healing as Technological Competence}

A second theme of the technological anecdote evident in medical dramas equates healing with technological competence. Institutions train and legitimate professionals and sort them into rigid hierarchies, depending on their skills in the language and practices of technology. The more complex and esoteric terminology and techniques are the prerogative of those at the top of the hierarchy, descending to those at the bottom who carry out orders and empty bedpans. Medical dramas occa- 
sionally challenge these hierarchies or value systems but overall serve to support the legitimacy of technological competence as the essence of healing. "Call the medical journals," stated an $E R$ physician, "we successfully completed an aortal by-thermal bypass in two hours and twenty minutes." In such small moments, medical dramas support the assumption that it is the technological elite that become heros of modern medicine.

During an episode of $E R$, for example, an interaction between the chief of surgery and a resident characterizes the way medical competence is habitually constructed in medical dramas. The chief of surgery was performing a laparoscopy on an elderly patient and accidently cut his gastric artery-a deadly mistake. In the ensuing controversy surrounding this death, the following conversation took place between the surgeon and the resident:

Surgeon: My last act as chief of surgery is to resign.

Resident: It was just one incident.

Surgeon: When I saw you in the trauma today, the passion that you exhibited-its been years since I felt like that. I can't handle the pressure. I feel like I just don't belong here anymore. I was a great surgeon. What happened, what I allowed to happen, it just proves that I am not a very great man.

In this episode, the surgeon had to confront his inability to command cutting-edge technology. In the particular case leading up to his resignation, the surgeon was more concerned about his relationship with the patient than with his technological competence — a fact that made him less able to perform well, at least in his own eyes. On realizing that he was caring about the patients more than his control of the technology, this doctor removed himself from the profession. Although the script acknowledges the ongoing tension between technological processes and healing arts, its suggests that a practitioner's value is determined by his technological competence, however deficient he may be in humanity. Certainly, we are not suggesting that incompetent physicians should continue to perform surgical procedures, only that in the technological paradigm, usefulness is equated with the competence to perform technologically.

Based on the scripts of medical dramas, technological competence also assumes that these physicians trust technology rather than lived experience. In an episode of $E R$, a blind man is hit in the head in a car accident and subsequently regains his eyesight. After their initial examination of the available scientific evidence, the doctors conclude that the patient was never originally blind and believe he is running a "scam," even though the man (with his seeing eye dog) emphatically claimed otherwise. Several hours later, the patient loses his sight again. Further tests revealed a tumor compressing against his optical nerve. The following conversation took place between a neurologist and Dr. Benton: 
Neurologist: From the size of the tumor, it has been growing for years.

Dr. Benton: How do you account for his temporary sight? Could the head trauma have caused it?

Neurologist: Well, maybe it caused some visual memory.

Dr. Benton: No, trust me, this guy could see. I was there. I saw it.

Neurologist: I don't know what to tell you. From the medical evidence in front of me, this man hasn't seen anything for five or six years.

In this example, Dr. Benton wanted to rely on his own senses to explore an explanation for what he believed to be the case- that the patient could see. However, the neurologist was at best unwilling and at worst sarcastically resistant to Dr. Benton's explanation of what he saw. In this case, the X-ray carried more medical clout than the visual observation of another doctor, reinforcing again that technological competence requires one to distrust experience and emotion. Belief in anything other than technology raises questions about competence.

In an episode of Chicago Hope, Dr. Kate Austin was demonstrating the use of a newly installed electromedical resourcing (EMR) system worth over $\$ 250,000$. The system was described by Dr. Austin as

The wave of the future. This system will make medical treatment so cost effective it will pay for itself ... . All you gotta do is feed it the patient's vitals and lab results and it gives you a complete diagnosis, a treatment plan, surgical recommendations, risk stratifications, everything.

Although Dr. Austin had opted to perform heart surgery on a patient, the EMR system recommended using beta blockers instead—something directly opposite to Dr. Austin's recommendation. The following conversation took place between Dr. Austin and the chief of staff about the EMR:

Dr. Austin: This isn't right. My patient has a thoracic aneurism that is 4.5 centimeters in diameter.

Chief of Staff: [reading printout] Beta blockers, surgery not recommended. What's wrong with that?

Dr. Austin: I've already scheduled surgery for in the morning - the exact opposite of this diagnosis. So, how do I know which one of us is right?

Chief of Staff: I don't know but for 250 grand my money is on the computer.

Dr. Austin ended up following the advice provided by the EMR system. In this case, Dr. Austin relied on technology rather than her own insights as a highly experienced surgeon. It is interesting to note that the man arrested following the beta-blocker treatment and surgery became the only option. The tension between 
insight and instrumentation was resolved on the side of insight, yet Austin never raised the question of the ultimate value of the EMR system and its displacement of insight and experience. In this script, technology again appears to be open to challenge but is ultimately reinforced as the paradigm of medical treatment.

Guided by the technological imperative, the earlier examples from Chicago $H o p e$ and $E R$ champion competent creators and users of technology, thereby reinforcing the hierarchies, social relations, and patterns of interactions within medical institutions. Healing is framed in the power of technology, in the belief that it supplants and substitutes for human emotion and insight. If on rare occasions human insight seems clearer and more accurate than technological knowledge, that occasion is merely the exception that proves the rule. This theme complements and strengthens the first. If nature is suspect, then competencies based in nature-emotion, intuition, insight-must likewise be suspect. In the battle against nature, competence must be technological.

\section{Alternative Philosophies of Medicine are Marginalized}

Throughout the past decade, Americans have begun to rediscover healing traditions not guided by allopathic philosophies. However, the conflation of technological medicine with healing means that any alternative approach is forced to work within the language, structures, assumptions, and expectations of technology. Terms like alternative, unconventional, and unorthodox are used to refer to such practices, thereby defining these systems of healing as "out of the ordinary" and therefore "of less value than" technological medicine. Alternative practitioners and techniques are suspect because they have not been legitimated by the standards of technological medicine. Results are judged by the criteria of technological-scientific inquiry, although the goals may be different (i.e., holistic integration rather than technological intervention).

Medical dramas reflect and reinforce the marginalizing powers of technological medicine. The tension between technology and its alternatives is displayed at several levels, not only in the language used to define alternatives but also by challenging the legitimacy of alternative practitioners. At every level, technological medicine works to marginalize alternatives and thus reinforce the dominance of technology. According to the doctors of Chicago Hope and ER, for example, holistic medicine appears to be equated with unscientific methods of diagnosis and treatment. Furthermore, such unorthodox therapies are viewed as posing a threat to public health and certainly are not considered standard medical practice. As said by one doctor on Chicago Hope, "the only thing herbs are good for is marinading chicken."

Medical dramas often portray the tension created when alternative and "real" doctors come together to try and counsel patients. One episode of Chicago Hope included a storyline in which an unborn child was at risk of death after only 24 weeks of pregnancy. The mother and father of the child had been attempting to conceive for 
several years and had little luck with standard medical treatments. A physical therapist in the hospital was trained to use acupuncture to facilitate fertility. As the couple faced medical problems with their unborn child, they wanted to include the therapist in decision making because she had treated the couple for infertility. As the therapist and Dr. Kate Austin attempted to negotiate roles with each other in terms of offering medical advice, tension developed. The following interaction illustrates the distress created as Dr. Austin confronted the acupuncture therapist:

Dr. Austin: You are out of line here Karen .... I agreed to let you be a part of this, but you can't influence these people's decision.

Karen: You don't have a clear answer [about the benefits of surgery] — why can't I weigh in?

Dr. Austin: Because you are not a doctor. You are giving her medical advice and you are not qualified — this isn't voodoo this is medicine .... You either leave these people alone and let them make their own decision or your involvement in this case is over.

After this interaction, the couple decided on in-vitro surgical intervention to save their child. After a successful surgery, the following interaction occurred between Dr. Austin and Karen, the acupuncture therapist:

Karen: Kate, thanks. You did great work.

Dr. Austin: I just wish we could have given them more time in-utero.

Karen: The baby is strong - it has a good chance of surviving.

Dr. Austin: Why do you insist on believing in things you have no evidence of?

Karen: Why do you insist on not believing? You know, we are not that different. You trust science-I trust something else. I believe that the truth has a way of gathering energy and we have to be open to seeing it.

Dr. Austin: Oh please. What nonsense .... You are a very scary person.

As proves to be the case in this example, legitimate technological practitioners usually have the last word, exhibiting skepticism at best and ridicule at worst. Therefore, health professionals espousing alternative therapies may be included in scripts, but are clearly portrayed as "fringe" elements of the medical profession.

This theme supports and extends the previous two themes. Although the first excludes nature, the second legitimates only those within the technological paradigm. It follows, then, that other forms of healing are suspect and their practitioners firmly defined as "outsiders." For instance, in the episode of Chicago Hope in which the two doctors were attending lamaze class, an interaction between Dr. Kronk and the lamaze instructor ended with Dr. Kronk stating, "Do you have any 
idea what you are talking about? I mean, you are not a real doctor are you?" In this example it seems clear that medical dramas reinforce the legitimate hierarchies among health care providers, defining those associated with technological medicine as "real," with others relegated to the margin and viewed with skepticism or even denounced as fraudulent.

\section{DISCUSSION}

Taken together, these three themes comprising the anecdote of technology are interlocking and mutually supporting ways of reinforcing technology as equivalent to medicine. The first theme, the opposition of technology and nature, has profound consequences that can only be briefly discussed here. In this "war" metaphor, nature is the enemy, a threat to the victory of technology. The drama consists of the ongoing struggle between technology and nature, as technology seeks to overcome nature and win, patient by patient, battle by battle. Technology, of course, expects to lose some battles, but the anecdote provides assurance that it will eventually prevail (i.e., provide a cure for every challenge of its enemy, nature). The dilemma, however, is that human emotion resides in nature, thus is a member of the enemy camp. Emotions interfere with the rational, logical, scientific decision-making processes that support technological interventions. Yet, technology ironically seems to require emotional commitment for its success, at least from the patients - they must "fight" and must "want to live." This dilemma remains an ongoing problem for technological medicine, and as such is frequently reflected in medical dramas.

The second theme, the reduction of healing to technological competence, reinforces the first in that the natural (i.e., emotional) qualities that make a good medical professional, qualities such as integrity or empathy, are silenced as technological competence is defined as the essence of professional competence. Those who display full command of the technological procedures, not those who are able to connect emotionally with patients, are invested with authority in the medical world. Emotions are implicitly marginalized as inconsequential to the medical encounter. Again, we see the paradox of emotions as technological medicine simultaneously devalues emotions and yet recognizes their power to challenge technology.

The third theme equates technological medicine with legitimate medicine, thereby marginalizing any other form of treatment. Other systems must be legitimated and discussed in the language of technological medicine, a test they are certain to fail because they may have vastly different definitions of health as well as different orientations to treatment. Here again, emotions intrude as many alternate systems are predicated on the unity of body, mind, and spirit and seek to employ emotions and the spiritual as allies in the healing arts.

The three themes of the anecdote in prime-time medical dramas intertwine to cement the technological imperative. The apparent openness of the scripting is seductive. On the surface, plots often appear to be receptive to nontechnological op- 
tions of treatment, initially implying that there are two different but equal choices available to patients and practitioners. As the drama proceeds, however, the dominance of technological medicine is implicitly as well as explicitly reinforced, visually and verbally. In the end, what appears to be a choice is no decision at all. Technology can be trusted, whereas natural remedies, although perhaps well meaning, cannot be trusted. They have not been tried and proven, they are not "under control" as is technology. Therefore, the discussion serves to silence alternative practices, sometimes by ignoring their presence, but increasingly by exploring options and ultimately judging them to be inferior to the technological imperative.

All three themes of the anecdote illustrate the paradox of emotions in the technological paradigm. Recent erosion of the public's faith in technological medicine has occurred, in part, because the system has alienated nature and displaced human emotion (Loustaunau \& Sobo, 1997). Recognition of this dilemma has begun to filter into medical dramas. Emotions are simultaneously the greatest threat and greatest promise for technological medicine. If technological medicine can maintain its emphasis on developing and delivering the best that technology has to offer and at the same time work with rather than against nature, value emotional competence as well as technological competence, and acknowledge the power of the mind and spirit in the healing process, those involved can experience the best of both worlds.

This article provides one reading on medical dramas. Future inquiry is needed to investigate how various audiences negotiate unique readings with the texts in the differentiated contexts in which they act in their everyday lives. Audience-centered research, with its emphasis on "the meeting place between television's discourse and television viewers" (Allen, 1992, p. 101), has potential to yield understandings of how readers interpret and derive pleasure from the texts in question. One audience in particular that deserves our attention is future health care providers. In her recent book on becoming a doctor at Harvard Medical School, Ellen Lerner Rothman (1999) discussed the loyalty her class experienced toward $E R$ and shared how students would measure their own newly acquired knowledge and skills against the discourse of $E R$ :

Through the $E R$ physicians, residents, and medical students, my classmates and I explored who we wanted to be and what we were afraid we might become. We developed a paradigm for how we wanted to respond to our patients and explored how we would feel if we were unable to uphold it. (p. 26)

Prior to ever entering work relationships, we have developed certain beliefs and expectations about how people communicate and interact in job settings (Jablin \& Krone, 1994). Media institutions, among other socializing agents, cumulatively affect our vocational development and perceptions of organizational life. Although Rothman's (1999) anecdotal evidence represents only one person's story about the powerful influence of $E R$, it suggests the future exploration of prime-time medical 
dramas from an audience-centered and socialization standpoint as a fruitful avenue of inquiry.

Underscoring our analysis is the assumption that critical cultural research designs represent a viable alternative for health communication researchers. The field of research generally incorporated under the rubric of health communication is dominated by the "effects" model in which communication theory is often applied to health care settings to overcome communication difficulties and improve physicians' and patients' communication competencies (Lupton, 1994). Culture, in this tradition of research, is either largely ignored or narrowly discussed as creating potential challenges for physician-patient encounters due to clashing norms or linguistic problems (Tulloch \& Lupton,1997). By recognizing that individuals' understanding of health, illness, and disease are constructed as part of social and cultural practices, cultural critics have much to offer health communication. Critics can examine the sociocultural constructions of health, illness, and disease as they are produced and reproduced in a number of sites (including TV texts such as medical dramas) to highlight the tacit understandings, assumptions, and belief systems underlying social structures and social actions.

Medical dramas have become important arenas for the creation and circulation of knowledge and experience about health, disease, and health care delivery. Televised fictional health care portrayals convey value-laden messages, the meanings of which contribute to the development of an ideological construction of our medical establishment. Throughout our analysis, medical dramas were treated as texts that serve as points of struggle or tension over meanings, particularly in relation to the role of technology in the delivery of health care. In sum, the discourse of medical dramas frames science, technology, and medical progress in such a way as to reinforce the ideology of the technological society. Both Chicago Hope and ER presume a future in which technology is a key element in the social matrix of medicine, yet both also provide openings, however slight, for change.

\section{ACKNOWLEDGMENTS}

We both contributed equally to the preparation of this article. Many thanks for the reviewers' helpful comments as well as the feedback from Deborah Japp and Brian Scott Titsworth.

\section{REFERENCES}

Allen, R. (1992). Channels of discourse, reassembled: Television and contemporary (2nd ed.). Chapel Hill: University of North Carolina Press.

Barnes, E., \& McDowell, J. (1998, June, 1). How good is ER's Rx? Time, 151, 74.

Brummett, B. (1984). Burke's representative anecdote as a method in media criticism. Critical Studies in Mass Communication, 1, 161-176. 
Burke, K. (1969). A grammar of motives. Berkeley: University of California Press.

Clarke, J. (1992). Cancer, heart disease, and AIDS: What do the media tell us about these diseases? Health Communication, 4, 105-120.

Glick, E., Berkanovic, E., Stone, K., Ibarra, L., Jones, M. L., Rosen, B., Schreibman, M., Gordon, L., Minassian, L., \& Richardes, D. (1998). Health education goes Hollywood: Working with prime-time and daytime entertainment television for immunization promotion. Journal of Health Communication, 3, 263-282.

Goodwin, J. S. (1999). Geriatrics and the limits of modern medicine. New England Journal of Medicine, $340,1283-1285$.

Jablin, F., \& Krone, K. (1994). Task/work relationships: A life-span perspective. In M. L. Knapp \& G. R. Miller (Eds.), Handbook of interpersonal communication (pp. 621-675). Thousand Oaks, CA: Sage.

Japp, P. (1991). Gender and work in the 1980s: Television's working women as displaced persons. Women's Studies in Communication, 14(1), 49-74.

Larson, M. (1991). Health related messages embedded in prime-time television entertainment. Health Communication, 3, 175-184.

Loustaunau, M. O., \& Sobo, E. J. (1997). The cultural context of health, illness, and medicine. Westport, CT: Bergin \& Garvey.

Lupton, D. (1994). Toward the development of critical health communication praxis. Health Communication, 6, 55-67.

McGinley, L. (1998, April 23). Moderate cost seen in boosting patients' rights. The Wall Street Journal, pp. B12, B14.

Parrott, R. (1996). Advocate or adversary? The self-reflective roles of media messages for health. Critical Studies in Mass Communication, 13, 266-278.

Pfau, M., Mullen, J., \& Garow, K. (1995). The influence of television viewing on public perceptions of physicians. Journal of Broadcast \& Electronic Media, 39, 441-458.

Rothman, E. L. (1999). White coat: Becoming a doctor at Harvard Medical School. New York: Morrow.

Sharf, B., \& Freimuth, V. (1993). The construction of illness on entertainment television: Coping with cancer on Thirtysomething. Health Communication, 5, 141-160.

Storey, J. (1993). An introductory guide to cultural theory and popular culture. Athens: The University of Georgia Press.

Treichler, P. A. (1989). What definitions do: Childbirth, cultural crisis, and the challenge to medical discourse. In B. Derwin, L. Grossberg, B. O'Keefe, \& E. Wartella (Eds.), Rethinking communication (Vol. 2, pp. 424-453). Newbury Park, CA: Sage.

Tulloch, J., \& Lupton, D. (1997). Television, AIDS, and risk: A cultural studies approach to health communication. St. Leonards, Australia: Allyn \& Unwin.

Turow, J., \& Coe, L. (1985). Curing television's ills: The portrayal of health care. Journal of Communication, 36-51.

U.S. Department of Health and Human Services. (1982). Television and behavior: Ten years of scientific progress and implications for the eighties. (DHHS Publication No. HE 20.8102:T 23/2/v.1-2). Washington, DC: U.S. Government Printing Office.

Vanderford, M. (1999). Television and religious values: A case study of ER and moral ambiguity. In F. Eigo (Ed.), Religious values at the threshold of the third millennium (pp. 33-73). Villanova, PA: Villanova University Press.

Zurawik, D. (1999, January 27). Doctor shows often a prescription for success. Lincoln Journal Star, p. 4D. 